Service social

\title{
Évaluation de signalement en utilisant l'approche de réseaux. Comment faire participer le milieu à la résolution de problème
}

\section{Richard Côté}

Volume 43, numéro 3, 1994

Intervention individualisée et empowerment

URI : https://id.erudit.org/iderudit/706667ar

DOI : https://doi.org/10.7202/706667ar

Aller au sommaire du numéro

Éditeur(s)

École de service social de l'Université Laval

ISSN

1708-1734 (numérique)

Découvrir la revue

Citer cet article

Côté, R. (1994). Évaluation de signalement en utilisant l'approche de réseaux. Comment faire participer le milieu à la résolution de problème. Service social, 43(3), 41-57. https://doi.org/10.7202/706667ar
Résumé de l'article

$\mathrm{Au}$ cours des dernières années, la Loi sur la protection de la jeunesse (LPJ) a fourni un cadre légal aux intervenants sociaux travaillant en CPEJ. Les intervenants se sentent souvent coincés entre leur rôle d'aidant et leur mandat légal. Cette situation est encore plus difficile en évaluation de signalement en protection de la jeunesse. Le présent article propose un modèle d'évaluation en contexte de protection, avec l'approche de réseaux, pour les jeunes qui présentent des troubles de comportement. Ce modèle vise à faire participer, de façon active, la famille dans le processus d'évaluation/orientation d'un signalement retenu. 


\section{Évaluation de signalement en utilisant l'approche de réseaux Comment faire participer le milieu à la résolution de problème}

Richard CÔTÉ Intervenant en évaluation / orientation, CPEJ de la Montérégie

Chargé de cours, Département de service social Université de Sherbroke

\section{INTRODUCTION}

La Loi sur la protection de la jeunesse (LPJ) a fourni, au fil des ans, un nouvel encadrement à l'intervenant social dans un cadre légal précis. La LPJ a divisé I'intervention en protection de la jeunesse en quatre grandes étapes.

\section{La réception et le traitement des signalements}

À la suite de renseignements reçus d'une ou de plusieurs personnes signalantes, l'intervenant évalue si les inquiétudes formulées par rapport à la situation d'un enfant peuvent compromettre la sécurité ou le développement de ce dernier. L'intervenant décide de la rétention ou non du signalement pour une investigation plus approfondie. 


\section{L'évaluation / orientation}

L'évaluation de la situation se fait sur le terrain par la rencontre des divers acteurs et par la prise de connaissance des faits liés à la situation signalée. Si l'intervenant peut démontrer que la sécurité et le développement de l'enfant sont compromis, il fait I'orientation du dossier par la proposition de mesures visant à corriger la situation de compromission. Ces mesures seront acceptées par les clients sur une base volontaire ou ordonnées par le juge de la Chambre de la jeunesse s'il y a opposition. Si la sécurité et le développement ne sont pas compromis ou que les faits sont non démontrés, l'intervenant ferme le dossier.

\section{La prise en charge du dossier}

L'intervenant du service de prise en charge voit à l'application des mesures pour mettre fin à la situation de compromission.

\section{La révision}

La révision périodique du dossier se fait durant la prise en charge de l'enfant. Elle permet de suivre l'évolution du dossier et de s'assurer que toutes les mesures sont appliquées pour mettre fin à la situation de compromission. À cette étape, I'intervenant ferme le dossier si la situation de compromission a disparu. Toutefois, si cette situation persiste, la prise en charge de l'enfant se poursuit.

Par ce cadre légal, le rôle du travailleur social comme agent de changement de la société peut facilement passer à un rôle de surveillance et de contrôle des familles. Notre intervention à l'intérieur de cette loi consiste à protéger les enfants qui vivent des situations qui compromettent leur sécurité et leur développement, à responsabiliser les parents à l'égard de la résolution des problèmes mis au jour et à offrir aux parents les services nécessaires pour régler la situation de compromission. La Loi souligne l'importance du rôle des parents en tant que premiers responsables face à l'enfant (art. 2.2, 2.3, 2.4)'. Bouchard $(1991)^{2}$ insiste sur le rôle prépondérant que doivent jouer les parents dans la résolution de leurs problèmes: "Après l'enfant lui-même, ce sont les parents qui sont les maîtres d'œuvre de son développement. À ce titre, ils ont des responsabilités, mais ils ont aussi besoin que la société les soutienne dans leur rôle ${ }^{3} »$. Harvey $(1991)^{4}$ ajoute: "Il faut développer le réflexe de chercher à associer systématiquement les clients aux décisions qui les concernent, dans une optique de responsabilisation ${ }^{5} »$. 
Dans les faits, le praticien prend la responsabilité de définir le problème selon sa perception de la situation de compromission et propose des mesures de protection en réponse aux difficultés de la famille. Cependant, ces solutions ne sont pas toujours adaptées à la réalité du client. Cette pratique tend à nier les capacités de ce dernier à l'égard de ses difficultés et à le déresponsabiliser. Nous croyons que le client doit être intimement lié à la résolution du problème, car il connaît son milieu, sa situation et les impacts sur son système. Le client doit jouer un rôle de premier plan dans le processus de résolution, car si le milieu fait partie de la source du problème, il fait aussi partie de la résolution. Selon Collins et Pancoast $(1976)^{6}$, il existe à l'intérieur du réseau des personnes qui ont la sensibilité et la compétence naturelle pour aider leurs proches. C'est vers eux que les personnes se tournent pour demander une assistance, souligne Pattison $(1975)^{7}$.

Dans cet article, nous présentons un modèle d'évaluation, par l'approche de réseaux, d'enfants qui présentent des troubles de comportement pour lesquels un signalement est retenu. Ce modèle vise à redonner à l'intervenant social son rôle d'aidant auprès de la clientèle. Il amène le client à développer et à utiliser ses forces et ses capacités ainsi que celles de son réseau primaire pour résoudre la situation problématique. Il vise à sensibiliser et à stimuler le réseau, afin d'aller chercher les ressources dont le client a besoin. Nous travaillons avec la famille ouverte sur son réseau de façon qu'elle fasse le travail avec son réseau. Ce modèle a été expérimenté dans les dossiers relatifs à l'article $38 \mathrm{~h}$ de la LPJ, mais nous croyons qu'il est applicable à d'autres problématiques.

Le modèle théorique s'inspire de l'approche de réseaux. Les autres approches d'intervention utilisées sont la thérapie de la réalité, I'approche systémique, l'intervention en situation de crise, la négociation et la concertation. Ces dernières servent d'outils $d^{\prime}$ intervention clinique et précisent certains concepts de l'intervention de réseaux en évaluation de signalements.

\section{LES POSTULATS}

- Le premier postulat est la confiance dans le potentiel et la richesse des capacités de la personne et de la famille à analyser, à agir et à régler la situation problématique.

Dans la situation difficile qu'elle vit avec son enfant qui présente des troubles de comportement, la famille éprouve un sentiment 
d'incompétence et de découragement devant les échecs de ses actions. L'intervention du Directeur de la protection de la jeunesse (DPJ) peut être perçue comme aidante pour certains parents qui ont signalé la situation; il n'en demeure pas moins que la famille se sent jugée et remise en question dans ses capacités parentales.

L'institution se reconnaît plus de pouvoir et de compétence devant la situation problématique et l'intervenant occupe une position d'expert. Les clients reconnaissent aussi ce pouvoir et cette position, ce qui diminue leurs initiatives et I'utilisation de leurs possibilités de prendre en charge leurs problèmes. Ce contexte encourage le client et son réseau à prendre une position d'hétéronomie face aux institutions.

Rousseau et Bélanger $(1985)^{8}$ définissent ainsi l'hétéronomie: «la tendance à remettre entre les mains d'un intervenant la responsabilité de définir, d'analyser et de solutionner les problèmes identifiés. L'hétéronomie correspond à la perte, chez les membres du réseau, du désir de définir et de construire leur propre environnement et de le modifier au besoin. C'est surtout la perte de confiance en la légitimité de ce désir.»

Le premier postulat consiste à nous démarquer, en tant qu'intervenants, de l'attitude d'experts que nous confèrent le client et I'institution. L'intervenant amène la famille et son réseau à mieux cerner leurs capacités; son rôle est de les soutenir dans cette démarche. L'intervenant se met à leur service par les moyens thérapeutiques et par ceux que lui confère son mandat légal.

\section{- Le deuxième postulat du modèle est la responsabilité. La famille est responsable d'elle-même.}

Chaque membre de la famille est impliqué dans la situation. La famille a sa part de responsabilité et est capable d'en assumer les conséquences. Les parents doivent prendre les moyens pour contrôler leur enfant. L'intervention, par ce second postulat, a une fonction de responsabilisation qui a pour but de faire participer la famille, de façon active, au processus de résolution de problème. Elle est basée sur le respect des valeurs et sur la compréhension de la réalité du client dans la mesure où celui-ci ne contrevient pas aux exigences de la société.

Dans les faits, au moment de l'intervention, les parents sont souvent en crise ou leurs comportements sont irresponsables. Ils n'ont plus accès à leurs ressources et ils éprouvent de la difficulté à se pren- 
dre en main. Ils démissionnent et s'en remettent aux institutions. L'intervenant se voit confier le mandat de non seulement régler le problème, mais de le faire sien. Face à la famille qui ne réussit pas par ses propres actions à rétablir la situation, il fait l'arrêt d'agir dans un premier temps (placement, contrat de comportement, etc.) pour offrir un répit à la famille et l'aider à se ressaisir. Par la suite, les interventions visent à redonner aux parents la responsabilité de la résolution du problème de leur enfant.

On peut percevoir le début de l'intervention comme une prise en charge du problème par l'intervenant, car celui-ci prend des décisions à court terme qui visent le changement et il s'implique directement auprès du jeune. L'intervenant prend le leadership de la situation problématique, ce qui peut sembler contredire le second postulat. Cette contradiction repose sur la confusion entre les conditions qui amènent les gens à décider de changer et la décision du changement. Ce n'est pas une prise en charge de la famille et du problème, mais plutôt une modification de certaines conditions où se trouve la famille. C'est donc une prise en charge du processus qui amène la famille à définir le problème et à activer ses ressources, comme le souligne Guy Ausloos (1990) ${ }^{9}$. La prise en charge de l'organisation des rencontres et des conditions qui faciliteront le changement se fait tout au long du processus. Elle peut demander une restructuration de la situation pour que le changement devienne possible.

Au cours de l'intervention, nous pouvons utiliser des moyens de contrôle (contrat de comportement) ou des moyens contraignants (placement en centre d'accueil), afin de limiter les actes irresponsables du client ou qui peuvent même être dangereux pour lui et les autres. Comme le mentionne Glasser $(1971)^{10}$, nous amenons le client à assumer la portée et la réalité de ses gestes (utilisation de la Chambre de la jeunesse, des policiers, séjours en centre d'accueil, contrôle scolaire, etc.). L'intervenant prend en charge le processus $\mathrm{d}^{\prime}$ activation du système pour que le client se prenne en main et cela, en collaboration avec les réseaux primaire et secondaire.

\section{- Le troisième postulat est l'aspect collectif du problème.}

La famille ayant des difficultés avec des enfants qui présentent des troubles de comportement ne vit pas en vase clos. Elle est entourée de sa famille élargie, de ses amis et de voisins qui sont des membres actifs en interaction avec la famille. La situation problématique est une manifestation d'un problème collectif. Ainsi, les 
difficultés comme la non-fréquentation scolaire, le défi à I'autorité parentale ou la consommation de drogues ne se développent jamais sans un environnement social qui favorise et parfois stimule activement le problème de comportement. La situation problématique est issue du milieu, la solution doit aussi venir de ce milieu.

Dans la réalité, le système a tendance à se refermer sur lui-même lorsqu'il vit des situations problématiques ou conflictuelles. La famille se sent ainsi isolée et incompétente devant son problème. Or, en tant qu'intervenant, nous avons tendance à nous centrer sur la personne qui présente des problèmes et sur sa famille. Par cette attitude, nous entrons dans le jeu du système familial. Le troisième postulat vise plutôt à sortir la famille de son isolement par une ouverture vers son réseau. Cette intervention demande au praticien une bonne connaissance de la famille et de son milieu, pour qu'il aide le système familial à exploiter les richesses existant dans son réseau.

\section{LES RÉSEAUX}

Guédon (1984) ${ }^{11}$ définit le réseau primaire comme un ensemble naturel de personnes qui ont des liens significatifs positifs ou négatifs entre elles dans la vie quotidienne (parents, amis, voisins, collègues de travail, etc.). Nous ajoutons à cette définition ce que nous nommons le réseau primaire actif, qui est constitué des membres du réseau primaire liés de plus près à la situation. Ces personnes sont vues par la famille comme des membres potentiellement aidants et souvent au courant de la situation. Ils peuvent être disponibles à court terme ou ils participent déjà au soutien et aident la famille.

Le réseau secondaire formel est formé par les institutions sociales qui ont une existence officielle. Ce sont des organismes structurés et organisés qui répondent à des besoins. Ils ont des fonctions spécifiques et fournissent des services particuliers (CPEJ, CLSC, hôpitaux, etc.).

Le réseau secondaire informel est constitué de groupes ou $\mathrm{d}^{\prime}$ associations qui ont un caractère moins officiel. Ceux-ci sont créés par des individus pour répondre à des besoins précis ou pour fournir des services particuliers, tels que les regroupements de locataires, les réseaux de gardiennage, etc. Ces réseaux informels sont moins structurés et plus éphémères que les institutions. Toutefois, ils ont la qualité d'être plus accessibles et plus adaptés aux besoins pour lesquels ils ont été créés. 


\section{LES OBJECTIFS DE L'INTERVENTION}

Les objectifs du modèle d'évaluation de signalement de réseaux sont d'évaluer le signalement retenu de façon à amener l'individualisation du problème signalé vers une collectivisation de la situation. De plus, le modèle vise à ce que l'attitude hétéronome montrée par la famille devant ses difficultés évolue vers une conduite autonome.

Ce modèle d'évaluation de signalement de réseaux se différencie de l'approche de réseaux sur trois points:

1) Il tient compte de l'individualité de la famille et de chacun de ses membres à l'intérieur de son milieu de vie. Le modèle d'évaluation de signalement de réseaux cherche à saisir comment la famille peut agir avec son collectif et ce que la famille peut aller chercher et apporter à son réseau afin d'améliorer sa propre situation dans un court laps de temps.

2) À cause du contexte de travail d'évaluation/orientation de signalements (intervention à court terme), l'intervention auprès de la famille est faite en fonction du réseau sans intervenir directement auprès de celui-ci. Elle vise donc à sensibiliser et à motiver la famille à se servir de son réseau pour qu'il l'aide, plutôt que ce soit l'intervenant qui fasse une démarche vers le réseau.

3) II semble ressortir des écrits sur l'intervention de réseaux que I'on doive amener la famille à être totalement autonome. Notre modèle d'évaluation de signalement de réseaux vise donc à amener la famille, qui était dans une situation hétéronome par rapport à l'institution, à atteindre une autonomie en ce qui a trait à son problème tout en gardant une hétéronomie des services que seules les institutions peuvent lui donner (les services de centre d'accueil, de thérapie, de groupe de thérapie et les moyens logistiques de l'institution). L'utilisation du réseau secondaire formel est parfois nécessaire pour la famille, afin de I'aider à atteindre ce que nous appelons une "hétéronomie autonome ». Cela se fait de deux façons. En premier lieu, la famille utilise de manière consciente et stratégique les services liés au problème offerts par les institutions. Ensuite, l'intervenant dans son rôle d'accompagnateur stimulera la famille, afin que celle-ci joue un rôle actif dans le processus de changement. II vise à ce que la famille n'ait pas une attitude hétéronome à l'égard de l'institution et de ses services, mais qu'elle constitue un apport important dans le processus d'orientation du dossier. Que la famille s'implique directement comme participant et non comme spectateur à l'intérieur de la démarche. 


\section{LES ATTITUDES DE L'INTERVENANT}

Nous avons un mandat légal en protection de la jeunesse. Notre attitude à l'égard du client détermine souvent le type de collaboration que nous aurons avec la famille durant l'évaluation du signalement. Grâce au modèle d'évaluation de signalement de réseaux, nous avons développé un ensemble d'attitudes que l'intervenant applique tout au long de l'intervention.

L'ouverture existentielle envers le client et les institutions pour qui et avec qui nous travaillons s'avère une attitude des plus importantes. Elle permet la libre et saine circulation de l'information, le respect des valeurs et du mode de vie et l'expression des sentiments et des réalités de chacun. Toutefois, dans un contexte d'intervention P.J. dans les dossiers de jeunes qui présentent des troubles de comportement, nous devons être directifs avec le client. L'ouverture existentielle se situe sur le plan de l'écoute et de I'empathie par rapport à la réalité du client. Le cadre temporel de l'intervention en évaluation de signalements est cependant limité. En effet, les comportements difficiles de I'adolescent demandent une intervention rapide et nous ne pouvons suivre le rythme de l'enfant pour l'amener à faire une prise de conscience et à régler la situation. Dans ce contexte d'intervention, nous utilisons l'ouverture existentielle disciplinée, $c^{\prime}$ est-à-dire que nous nous montrons ouverts à ce que le client vit et nous dit. Cependant, nous filtrons l'information en tenant compte de notre mandat, du rôle que nous jouons et surtout du but visé par notre intervention. Si nous attendons que l'adolescent soit prêt à faire des changements, il fuira la situation, d'où l'importance d'encadrer rapidement l'intervention.

La cohérence (discipline) de l'intervenant est une attitude importante. Avec des jeunes qui présentant des troubles de comportement sérieux et des parents qui sont souvent dépassés par la situation, nous devons avoir une attitude cohérente et structurée. Cette attitude est nécessaire afin d'encadrer ces jeunes et de rétablir un fonctionnement satisfaisant qui permette le développement de tous les membres de la famille. Souvent, le jeune est en désorganisation par rapport aux normes de la société et nous devons faire des arrêts d'agir. La cohérence aide à responsabiliser le jeune et à lui montrer qu'il ne peut plus tout décider de lui-même sans tenir compte de l'autorité parentale et sociale. Nous devons être assez forts pour soutenir notre action malgré les tentatives du client pour nous déséquilibrer et assez structurés pour donner des directives qui vont encadrer le jeune et sa famille. Nous devons donc être cohérents, prêcher par l'exemple, être responsables et disciplinés dans nos actions et dans ce que nous 
disons au jeune et à sa famille. Cette attitude est complémentaire à celle de l'écoute et de l'empathie.

Ces attitudes sont inspirées des idées énoncées par Carlos Castaneda (1985) ${ }^{12}$, qui mentionne l'importance d'être des guerriers pour éviter d'être manipulés de façon constante par des forces qui nous entourent (I'adolescent, la famille, nos collègues, nos patrons, etc.). Selon cet auteur, nous ne devons pas avoir seulement des principes, mais des stratégies, afin d'être fidèles à nos postulats. Les quatre grandes stratégies sont:

1. le contrôle de soi: contrôler certaines réactions internes en fonction du but visé ;

2. la discipline: faire des gestes tels que nous voulons qu'ils soient faits ;

3. I'endurance: attendre sans précipitation, sans anxiété, avec une satisfaction intérieure et avec simplicité ;

4. le sens du minutage: agir au moment stratégique dans le but de faire des interventions le plus efficaces possible. Les trois premières stratégies servent à construire et à préparer l'intervention, ce qui permet de faire des interventions précises de déséquilibre afin d'atteindre le changement.

\section{MÉTHODE D'INTERVENTION}

La méthode utilisée pour appliquer le modèle d'évaluation de signalement de réseaux est la suivante. La première étape est la lecture du signalement et du dossier. À cette première lecture, nous analysons de façon systémique la situation, en tenant compte de la forme de relation, de communication et de structure qui existe à l'intérieur du système familial. Nous procédons à une première identification du réseau qui entoure la famille. Nous considérons dès le départ la personne signalante comme un membre du réseau primaire actif, quelqu'un qui peut être potentiellement aidant. Ces premières données servent à faire une première représentation graphique de la famille et de son réseau. Nous émettons des hypothèses sur les règles de la famille, le type de communication et les frontières de la famille ainsi que le type d'interrelations qui existent à l'intérieur du réseau.

Ensuite, nous rencontrons successivement I'adolescent et son ou ses parents. L'entrevue peut se dérouler à l'école, au poste de police ou à la maison. Cette entrevue fait ressortir les divergences, 
les types de communication existants et les rôles des membres de la famille, les règles et les problèmes principaux dans une atmosphère moins conflictuelle que lorsque tous les membres de la famille sont réunis. Nous sommes à la période de la collecte de données et la rencontre individuelle permet souvent une expression plus facile des sentiments et des faits sans qu'il y ait une confrontation directe entre I'adolescent et ses parents. Par la suite, nous rencontrons l'enfant et ses parents pour faire le point et établir un mode $d^{\prime}$ intervention. À cette première rencontre, il est important de créer un espoir de résolution du problème et de répondre au besoin immédiat pour rétablir un certain équilibre dans la famille.

La deuxième rencontre se déroule avec toute la famille. Nous nous penchons alors sur les conditions socio-économiques de la famille, son environnement, ses conditions matérielles et son réseau. L'utilisation d'un questionnaire de réseaux permet de déterminer de façon claire les éléments potentiels du réseau qui peuvent aider et soutenir la famille. Son utilisation se fait de façon informelle et davantage sous forme d'échanges entre la famille et l'intervenant. À cette rencontre, nous identifions le réseau avec la famille et nous suggérons de communiquer avec certains membres du réseau et de les rencontrer. Nous commençons à remettre la responsabilité à la famille en ce qui a trait aux décisions et aux interventions à entreprendre. À cette rencontre, nous pouvons généralement décréter si la sécurité et le développement du jeune sont compromis en vertu de l'article 38 de la Loi sur la protection de la jeunesse, ce qui met fin à l'étape d'évaluation et permet de passer à la phase $d^{\prime}$ orientation du dossier ${ }^{13}$. Nous respectons ainsi le but de chaque étape administrative de notre dossier tout en poursuivant l'intervention clinique auprès de la famille et en continuant le processus d'accompagnement dans la démarche de résolution du problème.

Les autres rencontres visent à terminer I'orientation du dossier avec tous les membres de la famille et parfois avec des membres du réseau. Elles servent à évaluer le processus de résolution du problème, à compléter l'analyse en se servant des nouveaux éléments qui sont apparus, à savoir comment se sont déroulés les contacts avec les membres du réseau depuis la deuxième rencontre, et à connaître les résultats de ces rencontres. Elles permettent aussi d'évaluer l'impact du processus d'intervention sur la collectivisation du problème.

À l'étape de la prise de décision sur l'orientation du dossier, le praticien, la famille et parfois certains membres du réseau évaluent les mesures à prendre pour fermer le dossier ou le garder ouvert après 
avoir pris une décision d'intervention terminale, selon la définition du rapport Harvey ${ }^{14}$, si nous considérons qu'une intervention à court terme est suffisante. Si nous gardons le dossier ouvert, est-ce en vertu de mesures volontaires ou sous judiciarisation? Selon le cas, nous élaborons le contenu des mesures qui apparaîtront sur le formulaire des mesures volontaires ou qui seront présentées à la Chambre de la jeunesse. Cette démarche se fait avec la famille à l'intérieur d'une communication ouverte et d'un processus clinique dans lequel la famille et son réseau sont engagés directement. Si nous décidons de fermer le dossier, nous vérifions avec la famille son réseau de soutien et d'entraide et les mécanismes qu'elle pourrait utiliser advenant une autre situation difficile.

Dans le modèle, d'abord et avant tout, nous ne cherchons pas seulement les problèmes, mais les ressources nécessaires aux interventions et les ressources du milieu dans un but de collectivisation du problème. Nous utilisons le réseau secondaire, car celui-ci est en relation directe avec la résolution de la situation. Dans certaines situations, le réseau secondaire peut devenir une arme persuasive comme outil clinique dans le processus de résolution de problème. Cela s'inscrit à l'intérieur d'une stratégie de responsabilisation et de restructuration nouvelle de la situation vécue par le client.

Au cours de l'accompagnement dans le processus de résolution, il peut arriver que nous intervenions avec des praticiens ou des professionnels de diverses institutions ou avec des membres de la famille qui sont impliqués dans la situation problématique. C'est entre les membres de ces institutions et de la famille qu'il faut éclaircir la situation et déterminer les rôles et les enjeux de chacun. L'action demande à ce moment une concertation ou une négociation. Cette action se fait de façon que chaque partie sorte gagnante sur quelques points sans perdre la face, c'est-à-dire qu'on négocie sans perdant ni gagnant. Elle doit également se faire en croyant en la possibilité de procéder collectivement pour résoudre le problème.

\section{Les limites}

La méthodologie de ce modèle d'évaluation de signalement de réseaux est surtout centrée sur la famille et il y a peu d'interactions directes avec le réseau. Cette limite vient du cadre d'intervention (évaluation P.J.) qui donne peu de temps pour prendre une décision sur la compromission de la sécurité et du développement de l'enfant ainsi que sur l'orientation du dossier. Nous évaluons si le réseau existe, s'il exerce une influence, s'il possède des ressources et de 
quelle façon nous allons les activer. Notre fonction ne nous permet pas d'aller plus loin. II est impossible de penser à impliquer totalement le réseau ou à changer la relation de la famille avec son réseau dans les limites de notre travail et en un si court laps de temps. Nous intervenons donc auprès de la famille qui interviendra avec et dans son réseau.

\section{COMMENTAIRES SUR LE MODÈLE}

\section{Applicabilité}

À la suite de l'expérimentation de notre modèle d'évaluation de signalement de réseaux depuis quelques années à l'intérieur de notre pratique, nous constatons que ce modèle est applicable à l'intérieur du cadre de travail en évaluation / orientation des signalements. Le but visé est atteint. La famille et son réseau se sensibilisent et réagissent, à court terme, par rapport à une situation problématique vécue par la famille, en développant leur autonomie avec l'aide de leur collectif. La famille et une partie de son réseau prennent conscience de leur force et de leur capacité d'analyse et de résolution du problème. Les membres de la famille se prennent en charge sans se couper des ressources.

Quant à la durée de l'intervention pour évaluer un signalement à l'aide de notre modèle de pratique, elle correspond bien au cadre proposé dans le programme de la protection de la jeunesse ${ }^{15}$. Ce programme suggère qu'une évaluation dure onze heures. Or, si l'on comptabilise le temps accordé à l'intervention, sans tenir compte des déplacements et de l'attente à la Chambre de la jeunesse, la période de temps proposée par le programme est dans l'ensemble respectée par le modèle de pratique. L'argument souvent apporté du manque de temps pour faire ce type de démarche ne tient donc pas. On se rend compte qu'un processus d'intervention qui, au départ, paraît plus long et plus exigeant en matière de temps et $d^{\prime}$ investissement demande en fait à peu près le même temps que la pratique traditionnelle en usage chez les praticiens. Cela rend le modèle tout à fait applicable dans le cadre administratif de la DPJ. De plus, il faut noter que les dossiers avec une durée d'intervention plus longue ont été fermés, ce qui constitue un gain, car les situations ont été peu signalées de nouveau. Les interventions sont faites à l'intérieur de l'étape de $I^{\prime}$ intervention terminale, ainsi que nous le propose Harvey ${ }^{16}$. 


\section{Effets pour le praticien}

Ce processus d'intervention apporte une grande satisfaction au praticien. L'intervenant social se sent moins isolé dans le mécanisme de résolution du problème. Il établit une relation de collaboration avec les clients qui se sentent compris, écoutés et faisant partie de la solution, ce qui tend à diminuer le stress chez l'intervenant qui ne porte plus seul le poids de la résolution du problème. En responsabilisant le client, en faisant un partage des responsabilités et en I'accompagnant dans son processus de résolution de problème, I'intervenant trouve une détente valable contre le burn-out. Ce modèle insiste sur le fait que ce sont le jeune et les parents qui ont le problème, et non I'intervenant. II met I'accent sur les capacités du client plus que sur le besoin d'aide.

\section{Obstacles}

Dans la conjoncture actuelle où tout est structuré, organisé, technocratisé, l'État semble omniprésent dans les différentes sphères de la vie des gens. Le gouvernement par ses nombreuses politiques cherche à exercer un certain contrôle. Sans nier l'importance et la nécessité de la LPJ, celle-ci laisse parfois peu de place aux initiatives ou aux innovations sur le plan de l'intervention. Notre modèle de pratique propose une latitude d'action et de décision au réseau primaire. Pour certains praticiens, cette façon d'intervenir va à l'encontre de notre mandat. Qui prendra les décisions, qui contrôlera la situation et qui protégera l'enfant? Ce rôle revient aux parents. Une fois que le signalement retenu s'avère fondé, notre intervention consiste à nous assurer que les parents reçoivent et utilisent les services dont ils ont besoin pour résoudre la situation de compromission. Nous activons le processus de prise en charge et d'utilisation des ressources de la famille et de son réseau dans le but de régler le problème. Notre rôle de praticien est un rôle d'accompagnateur et de stimulateur des ressources de la famille, et non seulement celui d'une instance décisionnelle. Cette façon d'intervenir peut donner l'impression que le praticien perd le contrôle du dossier, alors que nous remettons à la famille sa part de contrôle et de responsabilisation. Nous nous mettons à son service à l'intérieur des limites du mandat et des normes que la société exige. C'est à ce moment que nous exerçons un contrôle efficace sur ce qui est modifiable à l'intérieur de la situation. 


\section{Ouverture}

Ce modèle met en évidence le fait que la famille possède souvent plus de ressources que nous voulons bien I'admettre. Il demande de prendre le temps d'être attentif aux réalités socio-économiques des gens et non seulement de se centrer sur le problème signalé, ce qui amène une ouverture du système familial et une affiliation plus facile avec lui. Les membres du système parlent aisément de leur réalité, de leurs activités et de leurs réseaux lorsqu'on crée un lien avec eux et qu'on s'attarde à leur vécu. Par cette attitude, les clients se montrent ouverts à l'idée d'informer les personnes de leurs réseaux. La famille comprend bien son rôle et elle a une perception positive de ses capacités.

\section{Constats}

Une des faiblesses du modèle, attribuable au système structurel de l'institution, est la discontinuité. Les délais qui existent avant que le dossier soit attribué à un autre praticien pour la prise en charge du dossier entraînent en effet une période de latence pour la famille. Celle-ci n'a personne à qui s'adresser durant une période où les acquis demeurent fragiles et la situation risque alors de se détériorer.

Nous remarquons que dans les dossiers où le modèle d'évaluation de signalement de réseaux est appliqué, la famille et son réseau effectuent des changements qui répondent à leurs besoins immédiats. Pour certaines familles, ces changements sont suffisants. Pour d'autres familles, une démarche plus en profondeur avec leurs réseaux devient nécessaire. Nous constatons cette situation dans les dossiers fermés qui ont été signalés à nouveau ou dans les dossiers pris en charge où la démarche amorcée avec le réseau n'ayant pas été poursuivie, la famille revient souvent à une attitude hétéronome et à une certaine passivité à l'égard de ses difficultés.

\section{CONCLUSION}

L'application du modèle d'évaluation de signalement de réseaux dans le contexte d'évaluation / orientation de signalements en troubles de comportement en protection de la jeunesse permet d'établir les bases de l'intervention de réseaux et ainsi d'accélérer le processus d'intervention.

Notre relation et le lien que nous entretenons avec les clients ont changé. Notre rôle avec les clients est clair. Nous avons confiance 
dans les capacités des gens, en tenant compte de leurs limites et de leurs valeurs. Nous ne cherchons pas à aller au-delà de ce qu'ils recherchent. Nous ne leur imposons pas, de façon implicite, nos objectifs personnels. En bref, résoudre la situation problématique ne devient pas notre défi personnel, il est celui de l'enfant et de ses parents. Le cadre d'intervention de la loi est précis. Il vise à définir les besoins et les mesures pour mettre fin à la situation de compromission d'un signalement retenu et à consolider les acquis pour éviter que cela se reproduise. Nous essayons de ne pas aller au-delà de ce cadre pour ne pas faire de l'ingérence dans la famille. Nous fonctionnons au rythme des clients et selon leurs demandes à l'intérieur de notre mandat. Nous avons amélioré notre écoute et nous encourageons les initiatives de la famille même si elles sortent des normes établies, mais non du cadre légal. Cela se fait à l'intérieur d'une ouverture à la différence et d'une acceptation de celle-ci. De plus, le modèle permet d'appliquer la concertation, ce qui fait partie de la volonté des politiques gouvernementales actuelles. Les acquis de la famille demeurent souvent fragiles et demandent un soutien et une continuité sur le plan de l'intervention. Comme le préconise Bouchard $(1991)^{17}$, la concertation entre les organismes communautaires et gouvernementaux permet d'assurer la continuité d'aide et de soutien dont la famille a besoin. Toutefois, la concertation est loin d'être intégrée dans la pratique. Les résistances des institutions sont fortes, car chaque institution a une notion différente de ce qu'est ou doit être la concertation. Caron $(1987)^{18}$ souligne que pour établir une saine concertation il faut du temps. En cette période de coupure de crédits et de services (qualité totale à peu de frais) où le Ministère demande aux réseaux des services sociaux et de la santé de répondre aux besoins de la clientèle et de fermer les dossiers rapidement, croire que I'on peut faire de la concertation à l'intérieur d'un tel régime semble un leurre. Ce climat ajoute de la pression tant chez les administrateurs et les cadres que chez les intervenants et a pour effet de chercher à refiler les dossiers à un autre organisme plutôt que de stimuler le travail de collaboration et de concertation. Malgré cela, des praticiens et des cadres de différents organismes sociaux et de la santé demeurent ouverts à de nouvelles approches et sont prêts à travailler en concertation avec les institutions. 


\section{Notes}

1. Gouvernement du Québec, Loi sur la protection de la jeunesse.

2. C. Bouchard, Rapport du groupe de travail pour les jeunes, Un Québec fou de ses enfants. Québec, 1991.

3. Id., p. 19.

4. J. Harvey, La protection sur mesure: un projet collectif, Québec, 1991.

5. Id., p. 35.

6. G. Ouimet, «L'intervention de réseaux appliquée à des jeunes délinquants », Service social, vol. 32, $n^{\circ} 1-2,1983$.

7. Id.

8. R. Rousseau et M. Bélanger, Les outils d'intervention de réseaux, Montréal, 1985, p. 4.

9. G. Ausloos, De la compréhension à l'activation thérapeutique. Texte inédit, 1990.

10. W. Glasser, La thérapie par le réel. La reality therapy. Paris : EPI, 1971.

11. M.-C. Guédon, "Les réseaux sociaux», dans L'intervention de réseaux une pratique nouvelle. Montréal : France-Amérique.

12. C. Castaneda, Le feu du dedans. Paris: Gallimard, 1985.

13. J. Harvey, Rapport sur l'analyse des activités de RTS et d'E/O en protection de la jeunesse, 1988.

14. A. Lebon, Protocole d'intervention terminale en protection de la jeunesse, 1990.

15. CSSR, Programme de protection de la jeunesse, élément de "protection», 1986, p. 104.

16. CSSR, op. cit.

17. Id.

18. A. Caron, Recherche comparative sur les conditions de faisabilité de la concertation principalement au niveau du secteur jeunesse, Centraide Montréal, 1987. 


\section{Références bibliographiques}

AusLOOS, G. (1990). De la compréhension à l'activation thérapeutique. Texte inédit, $24 \mathrm{p}$.

BÉLANGER, M. et R. ROUSSEAu (1985). Les outils d'intervention de réseaux. Publié par le Groupe de recherche sur les pratiques de réseaux, $15 \mathrm{p}$.

CARON, A. (1987). Recherche comparative sur les conditions de faisabilité de la concertation principalement au niveau du secteur jeunesse. Centraide Montréal, Montréal, 139 p.

CAStaneda, C. (1985). Le feu du dedans. Paris: Gallimard.

CSSR (1986). Programme de protection de la jeunesse, élément de «protection», $108 \mathrm{p}$.

GLASSER, W. (1971). La thérapie par le réel. La reality therapy. Paris: EPI.

GOUVERNEMENT DU QUÉBEC (1988). Rapport sur l'analyse des activités de $R T S$ et d'E/O en protection de la jeunesse (Rapport Harvey). Québec: Ministère de la Santé et des Services sociaux.

GOUVERNEMENT DU QUÉBEC (1991). La protection sur mesure, un projet collectif. Rapport du groupe de travail sur I'application des mesures de protection de la jeunesse (rapport Harvey II). Québec: Ministère de la Santé et des Services sociaux.

GOUVERNEMENT DU QUÉBEC (1991). Un Québec fou de ses enfants. Rapport du groupe de travail pour les jeunes. Québec: Ministère de la Santé et des Services sociaux.

GuÉDON, M.-C. (1984). "Les réseaux sociaux», dans L'intervention de réseaux, une pratique nouvelle. Montréal: France-Amérique.

LeBON, A. (1990). Protocole d'intervention terminale en protection de la jeunesse, basée sur les travaux d'un groupe d'experts inter-CSS, dans les suites du rapport Harvey.

OUIMET, G. (1983). «Études du processus d'intervention de réseaux appliqué à des jeunes délinquants », Service social, vol. 32, n 1-2:186-194. 